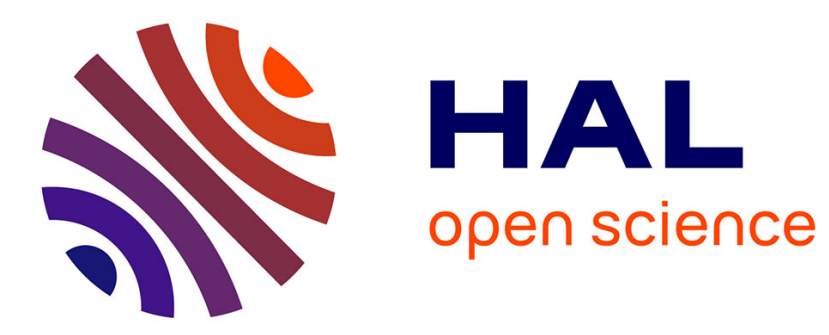

\title{
A reliability-based approach to determine the minimum detectable damage for statistical damage detection
}

\author{
Alexander Mendler, Michael Döhler, Carlos E Ventura
}

\section{To cite this version:}

Alexander Mendler, Michael Döhler, Carlos E Ventura. A reliability-based approach to determine the minimum detectable damage for statistical damage detection. Mechanical Systems and Signal Processing, 2021, 154, pp.107561. 10.1016/j.ymssp.2020.107561 . hal-03276728

\section{HAL Id: hal-03276728 \\ https://hal.inria.fr/hal-03276728}

Submitted on 2 Jul 2021

HAL is a multi-disciplinary open access archive for the deposit and dissemination of scientific research documents, whether they are published or not. The documents may come from teaching and research institutions in France or abroad, or from public or private research centers.
L'archive ouverte pluridisciplinaire HAL, est destinée au dépôt et à la diffusion de documents scientifiques de niveau recherche, publiés ou non, émanant des établissements d'enseignement et de recherche français ou étrangers, des laboratoires publics ou privés. 


\title{
A Reliability-based Approach to Determine the Minimum Detectable Damage for Statistical Damage Detection
}

\author{
Alexander Mendler ${ }^{a, b, *}$, Michael Döhler ${ }^{\mathrm{b}}$, Carlos E. Ventura ${ }^{\mathrm{a}}$ \\ ${ }^{a}$ University of British Columbia, CEME, 6250 Applied Science Lane, Vancouver BC, V6T 1Z4, Canada \\ ${ }^{b}$ Univ. Gustave Eiffel, Inria, COSYS-SII, I4S, Campus de Beaulieu, 35042 Rennes, France
}

\begin{abstract}
This paper derives a formula to determine the minimum detectable damage based on ambient vibration data. It is a key element to analyze which damage scenarios can be detected before a monitoring system is installed. For the analysis, vibration data from the reference structure as well as a finite element model are required. Minimum detectability is defined by adopting a code-based reliability concept that considers the probability of detection and the probability of false alarms. The results demonstrate that the minimum detectable damage depends on three elements: the uncertainty of the damage-sensitive feature (which decreases with increasing measurement duration), its sensitivity towards model-based design parameters, and the reliability requirements regarding the damage diagnosis results. The theory is developed for the stochastic subspace-based damage detection method but can be applied to any damage-sensitive feature provided its sensitivities and statistical properties can be characterized. For proof of concept, the minimum detectable change in stiffness and mass of a pin-supported beam are analyzed in a numerical and experimental study, respectively. The predictions with the developed approach appear to be accurate and robust to noise effects for both simulated and real data.
\end{abstract}

Keywords: Damage detectability, ambient vibrations, sensitivity, Fisher information, probability of detection, measurement duration

\section{Introduction}

Automated damage diagnosis is an integral part of structural health monitoring (SHM) [1-3]. With the availability of new technologies, the instrumentation of civil engineering structures is increasingly recognized as an economic advantage and has been established in numerous design codes around the world [4]. In particular, vibration-based approaches have been actively developed. Many methods that are based on modal parameter changes (i.e. changes in the natural frequencies, mode shapes, etc.) have transitioned

\footnotetext{
*Corresponding author; E-mail address: alexander.mendler@ubc.ca
} 
into engineering practice with applications in the monitoring of high-rise buildings, bridges, and offshore structures [5-7]. The basic premise is that damage will alter the stiffness, mass or damping properties of the structure, so it can be inferred based on the global system response. Output-only methods based on the assumption of ambient excitation are particularly interesting, as dynamic properties can be evaluated under normal operating conditions and without human interaction [8, 9]. However, they are often criticized because of the low signal-to-noise ratio, the low sensitivity to small and local damages, and the low reliability of the damage diagnosis [10]. Another problem is that most damage detection methods are set up based on data from the structure in the reference state, and during monitoring, it is unclear what damage scenarios can or cannot be detected. This makes it hard to fine-tune the instrumentation to be installed and convince decision-makers of the method's effectiveness. Motivated by this, a formula is derived in this paper that predicts the minimum detectable damage for ambient vibration monitoring. It relates changes in damagesensitive features to a degradation of engineering design parameters that are decisive for the structural health and safety. This way, the performance of the considered damage detection method can be assessed before damage occurs, which is a relevant asset before deploying SHM in practice. Ultimately, it links global vibration analysis to structural reliability analysis [11] and helps to evaluate the value of monitoring information in general [12]. For the derivation, multiple challenges related to ambient vibration testing have to be overcome.

The first challenge is to relate data-driven features to structural design for a physical interpretation of anomalies in the data. To link time histories, correlation functions, or derived damage-sensitive features to structural design parameters, additional information from finite element models is necessary. There are numerous ways to establish this link and a large group can be classified as dynamic model fitting methods. Herein, dynamic system properties are extracted from a model and fitted to quantities estimated based on data. In model updating, for example, the mass and stiffness matrix of a model is updated by confronting it with data-driven frequencies and mode shapes and minimizing the resulting modal force vector (or residual force) [13]. The subspace fitting method estimates the dynamic observability matrix from data and updates the model-based observability through linear regression [14, 15]. Another group of methods uses sensitivities to link features to structural parameters. In sensitivity-based model updating, for example, sensitivity matrices are used to map changes in natural frequencies and mode shapes onto structural parameters in the model [16-18]. Vice versa, sensitivity matrices can also be used to include physical information from numerical models in data-driven hypothesis tests that are directly applied to the damage-sensitive feature [19-21]. These sensitivities are a fundamental element in this paper.

The second challenge is that features computed from ambient vibrations are subject to statistical variability. Uncertainties are always present due to unknown excitation, measurement noise, and limited data, and they are amplified in records with weak structural signals and strong noise components. Additional uncertainties are introduced if environmental and operational conditions change (e.g. due to changes in 
operational loads [22] or ambient temperatures [8]), and if badly calibrated finite element models are employed for damage diagnosis [16]; however, such uncertainties are not addressed in this paper. The normal condition of the structure can be defined by evaluating confidence limits for the damage-sensitive feature based on training data [2]. Outliers can be highlighted by applying statistical tests that consider variations in the normal condition through the feature's covariance (e.g. the Mahalanobis distance [23], the Hotelling's $T^{2}$-test [24], the likelihood ratio [25], and others), where the test statistic is evaluated against a problemspecific threshold in control charts [26]. Following this logic, damage is detectable if it causes the damage indicator (i.e. the feature or the test statistic) to exceed the threshold value. There are various examples where detectability is quantified through a minimum deviation in natural frequencies $[27,28]$ or in test responses [24], but the approaches are empirical, problem-specific, and cannot be generalized, mainly because they lack a link between the damage indicators and structural parameters, as explained above. To further analyze the magnitude of the damage indicator as well as its uncertainty, a mathematical relation between its statistical distribution in the damaged state and the physical state of design parameters is necessary. One damage detection method that provides such a relation is the stochastic subspace-based damage detection (SSDD) $[22,25,29,30]$, which is used here as an example to demonstrate the computation of the minimum detectable damage.

Thirdly, the notion of minimum detectability implies that there is a certain level of reliability regarding the damage diagnosis result. The probability of detection $(P O D)$ is the most common measure to describe the quality of a damage diagnosis [31]. It quantifies the probability that a damaged structure is correctly classified as damaged. The POD typically depends on the amount of damage sustained and the relation is often described through so-called POD-curves. For example, when detecting surface-near cracks in metal through non-destructive field tests, the POD-curve can be plotted over the crack depth [31]. Another measure is the probability of false alarms (PFA), which quantifies how often a healthy structure is diagnosed as damaged although no damage is present. Typically, the safety threshold for the reference state is defined by the user based on an acceptable PFA, but since the safety threshold also affects the POD for a given damage, damage detectability involves both the PFA and the desired POD [32, 33]. Ultimately, empirical studies have shown that the reliability of the damage detection can be increased through an increased measurement duration [24], but no analytical relationship has been used to determine an appropriate measurement duration.

The developments for the minimum detectable damage in this paper consider the aforementioned aspects, i.e. the statistical variability of the damage-sensitive feature, its link to structural design parameters through FE models, and the required reliability of the damage detection results. In the framework of SSDD, the reference state is characterized through the probability density function (PDF) of the damage-sensitive feature and changes in this PDF are linked to model-based design parameters through the Fisher information $[20,21]$. Such design parameters include the material properties, cross-sectional values, or pre-stressing forces, which are decisive quantities for structural design, structural health, and thus, safety. Moreover, 
reliability considerations are incorporated including a user-defined PFA and POD, which can, for example, be chosen based on design standards, with reliability indices discussed in [6]. By combining the resulting equations with fundamental statistical approaches, a formula for the estimation of the minimum detectable change is derived, which also depends on the measurement duration. The approach is more universal than previous attempts, e.g. in [34, 35], because it is not restricted to specific types of structures, structural members, or monitoring parameters, and because it not only indicates whether or not damage is detectable but also quantifies the detectable damage in percent of the chosen design parameter. In other words, it is a key element to evaluate which structural changes can be detected before a SHM system is installed.

The paper is organized as follows: Section 2 gives a detailed problem statement for the analysis of the minimum detectable damages. Section 3 provides background information on the dynamic system models, the damage-sensitive feature, and the damage detection method. Section 4 proposes an approach to estimate the minimum detectable damage for this method, by employing additional information from a FE model, as well as theoretical proof that the prediction is valid for data-driven tests. For proof of concept, the methodology is applied to simulated and real data of a steel beam in Section 5, with a subsequent discussion in Section 6.

\section{Problem Statement}

Are vibration-based damage detection methods sensitive enough to detect small and local damages, in particular, if the excitation is unknown? One way to answer this question is to evaluate the minimum extent of damage that can be detected reliably in an arbitrary structural component, which is the approach taken in this paper.

To find this minimum detectable damage, damage has to be defined first. In structural engineering, damage is understood as a degradation of design parameters, such as, material constants or geometrical constants [2, 36], cross-sectional values or prestressing forces [37], or the mass-distribution [38]. A convenient way to parametrize these scenarios is to model the structure using FE software and user-define structural parameters, the change in which manifests damage. The effect of such parameter changes on the damagesensitive feature that is used for damage detection can be evaluated through sensitivity analyses. Since the feature is computed based on vibration data under unknown excitation and measurement noise, statistical uncertainties are introduced. Therefore, changes in the damage-sensitive feature are only indicative of damage if they are significant, in a statistical sense, meaning if they exceed a safety threshold value that was previously defined while training the algorithm based on reference data. In other words, the problem of evaluating the minimum detectable damage boils down to finding the minimum change in a model-based design parameter that, despite uncertainties due to noisy vibration data, allows for a reliable diagnosis.

Hence, the following aspects directly affect the minimum detectable damage: 
(a) Damage-sensitive feature and its sensitivity

(b) Signal-to-noise ratio

(c) Measurement duration

(d) Reliability requirements for the damage detection results

(i.e. probability of false alarms in the reference state, and the probability of detecting damages)

Maximum detectability is given for a high sensitivity of the feature, a high signal-to-noise ratio, a long measurement duration, and low requirements regarding the reliability. The interplay of all factors will be condensed into a formula for the minimum detectable damage on the example of the stochastic subspacebased damage detection method $[22,29]$. In the next section, some background on this method is given.

\section{Background on the Damage Detection Method}

\subsection{Dynamic System Models}

The vibrations of a linear and time-invariant mechanical system with $m$ degrees of freedom (DOF) can be modeled through the equation of motion

$$
\mathcal{M} \ddot{\mathbf{u}}(t)+\mathcal{C} \dot{\mathbf{u}}(t)+\mathcal{K} \mathbf{u}(t)=\mathbf{f}(t)
$$

including the mass, damping, and stiffness matrices $\mathcal{M}, \mathcal{C}, \mathcal{K} \in \mathbb{R}^{m \times m}$, as well as the displacement of the structure $\mathbf{u}(t) \in \mathbb{R}^{m}$ and its time derivatives under external forces $\mathbf{f}(t) \in \mathbb{R}^{m}$. Vibration measurements are typically available as displacements, velocities, or accelerations records. They can be described by the measurement vector

$$
\mathbf{y}(t)=\mathbf{L}_{d} \mathbf{u}(t)+\mathbf{L}_{v} \dot{\mathbf{u}}(t)+\mathbf{L}_{a} \ddot{\mathbf{u}}(t)+\mathbf{e}(t),
$$

where $\mathbf{L}_{d}, \mathbf{L}_{v}, \mathbf{L}_{a} \in\{0,1\}^{r \times m}$ are binary matrices that assign every measurement quantity from $r$ sensors to the corresponding channels in the measurement vector $\mathbf{y}(t) \in \mathbb{R}^{r}$, and $\mathbf{e}(t)$ is the measurement noise. For efficient signal processing, both equations are transferred into discrete-time $t=k \tau$ with the time step $\tau$ and the second order derivatives are substituted through the definition of a state vector $\mathbf{x}_{k} \in \mathbb{R}^{n}$ in the state space model [39]

$$
\left\{\begin{array}{rl}
\mathbf{x}_{k+1} & =\mathbf{A} \mathbf{x}_{k}+\mathbf{w}_{k} \\
\mathbf{y}_{k} & =\mathbf{C} \mathbf{x}_{k}+\mathbf{v}_{k}
\end{array} \quad \mathbf{x}_{k}=\left[\begin{array}{c}
\mathbf{u}(k \tau) \\
\dot{\mathbf{u}}(k \tau)
\end{array}\right]\right.
$$

where $\mathbf{y}_{k}=\mathbf{y}(k \tau) \in \mathbb{R}^{r}$ is the sampled output vector and $n=2 m$ is the system order. The state noise $\mathbf{w}_{k} \in \mathbb{R}^{n}$ and the output noise $\mathbf{v}_{k} \in \mathbb{R}^{r}$ include the unknown force term $\mathbf{f}$ and measurement noise $\mathbf{e}$, and are assumed to be white noise with zero mean. $\mathbf{A} \in \mathbb{R}^{n \times n}$ and $\mathbf{C} \in \mathbb{R}^{r \times n}$ are the state transition and the output matrices with

$$
\mathbf{A}=\exp \left(\left[\begin{array}{cc}
\mathbf{0} & \mathbf{I} \\
-\mathcal{M}^{-1} \mathcal{K} & -\mathcal{M}^{-1} \mathcal{C}
\end{array}\right] \tau\right), \quad \mathbf{C}=\left[\begin{array}{ll}
\mathbf{L}_{d}-\mathbf{L}_{a} \mathcal{M}^{-1} \mathcal{K} & \mathbf{L}_{v}-\mathbf{L}_{a} \mathcal{M}^{-1} \mathcal{C}
\end{array}\right]
$$


The classical solution to the eigenvalue problem $\mathbf{A} \boldsymbol{\Phi}=\boldsymbol{\Phi} \boldsymbol{\Lambda}$ yields the eigenvalues $\boldsymbol{\Lambda}=\operatorname{diag}\left(\left[\lambda_{1}, \ldots, \lambda_{n}\right]\right)$ of the discrete-time system and the observed eigenvectors $\mathbf{\Psi}=\mathbf{C \Phi}=\left[\begin{array}{lll}\boldsymbol{\psi}_{1} & \ldots & \boldsymbol{\psi}_{n}\end{array}\right]$.

\subsection{Damage-sensitive Feature}

The subspace-based damage-sensitive feature $[22,29]$ is formed based on the output covariances $\mathbf{R}_{i}=$ $E\left[\mathbf{y}_{k+i} \mathbf{y}_{k}^{T}\right]$, which evaluate the similarity of the signals at different sensor locations and time lags $i=$ $1, \ldots, p+q$, where $\min \{p r, q r\} \geq n$ with usually $p+1=q$. When arranged in a block Hankel matrix $\mathcal{H}$, the dynamic system matrices $\mathbf{A}$ and $\mathbf{C}$ from Eq. (4) can be retrieved from its column space through singular value decomposition (SVD), as it is done in stochastic subspace-based identification [40, 41]. The block Hankel matrix is, therefore, the first signal processing unit to capture the dynamic properties of a vibrating structure and the subspace decomposition leads to

$$
\mathcal{H}=\left[\begin{array}{cccc}
\mathbf{R}_{1} & \mathbf{R}_{2} & . \cdot & \mathbf{R}_{q} \\
\mathbf{R}_{2} & \mathbf{R}_{3} & . \cdot & \mathbf{R}_{q+1} \\
\cdot \cdot & . \cdot & . \cdot & . \cdot \\
\mathbf{R}_{p+1} & \mathbf{R}_{p+2} & . \cdot & \mathbf{R}_{p+q}
\end{array}\right]=\left[\begin{array}{ll}
\mathbf{S}_{1} & \mathbf{S}_{0}
\end{array}\right]\left[\begin{array}{cc}
\mathbf{D}_{1} & \mathbf{0} \\
\mathbf{0} & \mathbf{0}
\end{array}\right]\left[\begin{array}{c}
\mathbf{W}_{1}^{T} \\
\mathbf{W}_{0}^{T}
\end{array}\right] .
$$

The non-zero singular values in $\mathbf{D}_{1}$ are ranked in descending order and the corresponding singular vectors $\mathbf{S}_{1}$ are referred to as the column space. $\mathbf{S}_{0}$ defines the left null space which, in the following, is assumed to be extracted from the reference state. Due to the orthogonality of the two vector spaces, it holds that $\mathbf{S}_{0}^{T} \mathcal{H}=\mathbf{0}$ if and only if $\mathcal{H}$ corresponds to the reference state, since damage alters the dynamic properties in the block Hankel matrix. Hence, a damage-sensitive feature vector can be formed by confronting the block Hankel matrix estimate from incoming data with the left null space from the structure in the reference state. The resulting matrix can be condensed into a residual vector $\varepsilon$ through the vectorization operator

$$
\varepsilon=\operatorname{vec}\left(\mathbf{S}_{0}^{T} \hat{\mathcal{H}}\right)
$$

The hat symbol indicates that the Hankel matrix is calculated based on finite measurements of length $N$, containing the estimated output covariances $\hat{\mathbf{R}}_{i}=\frac{1}{N-i} \sum_{k=1}^{N-i} \mathbf{y}_{k+i} \mathbf{y}_{k}^{T}$. The feature $\boldsymbol{\varepsilon}$ is called residual vector in the following. Since it is computed from noisy vibration data under stochastic system excitation, it is subject to statistical uncertainties that have to be taken into account to allow for a reliable damage detection.

\subsection{Damage Detection Method}

A common approach to characterize a vibrating structure is the definition of a monitoring parameter vector $\boldsymbol{\theta}$ which is, for instance, a vector containing modal parameters or structural design parameters. The monitoring parameters in the reference state are assumed to be known and deterministic. During monitoring, the state of the parameter vector is unknown but a hypothesis can be made that it changes from its known 
reference state with $\boldsymbol{\theta}=\boldsymbol{\theta}^{0}$ to the damaged state with $\boldsymbol{\theta}=\boldsymbol{\theta}^{0}+\Delta \boldsymbol{\theta}$. The respective hypotheses in the framework of the asymptotic local approach [42] are

$$
\begin{array}{ll}
H_{0}: \boldsymbol{\theta}=\boldsymbol{\theta}^{0} & \text { (reference state) } \\
H_{1}: \boldsymbol{\theta}=\boldsymbol{\theta}^{0}+\boldsymbol{\delta} / \sqrt{N} & \text { (damaged state) }
\end{array}
$$

where $\boldsymbol{\delta}=\sqrt{N}\left(\boldsymbol{\theta}-\boldsymbol{\theta}^{0}\right)$. The definition of the change vector for statistical analysis $\boldsymbol{\delta}$ is for mathematical convenience, as it allows for the residual's distribution to be characterized in the following. It also implies that smaller changes in the monitoring parameters $\boldsymbol{\theta}$ can be detected with increasing data length, as $\Delta \boldsymbol{\theta}=$ $\boldsymbol{\theta}-\boldsymbol{\theta}^{0}=\boldsymbol{\delta} / \sqrt{N}$. Using the first-order Taylor approximation $E_{\boldsymbol{\theta}}[\varepsilon] \approx \mathcal{J}\left(\boldsymbol{\theta}-\boldsymbol{\theta}^{0}\right)$, the residual's deviation from the reference state can be parametrized, where

$$
\mathcal{J}=\left.\frac{\partial E_{\boldsymbol{\theta}}[\varepsilon]}{\partial \boldsymbol{\theta}}\right|_{\boldsymbol{\theta}=\boldsymbol{\theta}^{0}}
$$

is the first-order sensitivity matrix with respect to the monitoring parameter vector $\boldsymbol{\theta}$, and $\boldsymbol{E}_{\boldsymbol{\theta}}$ denotes the expected value of a variable calculated under $\boldsymbol{\theta}$. It can be shown that, after multiplication with the square root of the data length $\sqrt{N}$, the residual satisfies the central limit theorem (CLT) [25]

$$
\boldsymbol{\zeta}=\sqrt{N} \varepsilon \rightarrow \begin{cases}\mathcal{N}(\mathbf{0}, \boldsymbol{\Sigma}) & \text { under } H_{0} \\ \mathcal{N}(\mathcal{J} \boldsymbol{\delta}, \boldsymbol{\Sigma}) & \text { under } H_{1}\end{cases}
$$

for $N \rightarrow \infty$, meaning the distribution of $\boldsymbol{\zeta}$ is approximated by a Gaussian distribution. The asymptotic covariance matrix $\boldsymbol{\Sigma}$ describes the uncertainties in the damage-sensitive residual due to stochastic excitation and measurement noise, and it is identical under both hypotheses (7) [42]. Besides the damage-sensitive feature from Eq. (6), the framework described through the CLT from Eq. (9) can be applied to many other features [23, 43-45], some of which are robust to changes in the ambient excitation properties [22] or environmental conditions [19, 46]. Since the residual mean in Eq. (9) is zero in the reference state and different from zero in the damaged state, the damage detection problem ultimately boils down to monitoring the residual's mean vector.

To assess the significance of changes in the mean vector of the Gaussian residual, a statistical hypotheses test, namely the generalized likelihood ratio (GLR) test, is applied. With the GLR test, a decision is made whether the residual computed based on incoming vibration data is more likely to be the realization of the probability density function (PDF) corresponding to the structure in the reference or in the damaged state. Considering Eq. (9), the test statistic can be formulated as [25]

$$
t=\boldsymbol{\zeta}^{T} \boldsymbol{\Sigma}^{-1} \mathcal{J}\left(\mathcal{J}^{T} \boldsymbol{\Sigma}^{-1} \mathcal{J}\right)^{-1} \mathcal{J}^{T} \boldsymbol{\Sigma}^{-1} \boldsymbol{\zeta} \rightarrow\left\{\begin{array}{lll}
\chi^{2}(\nu, 0) & \text { under } & H_{0} \\
\chi^{2}(\nu, \lambda) & \text { under } & H_{1},
\end{array}\right.
$$




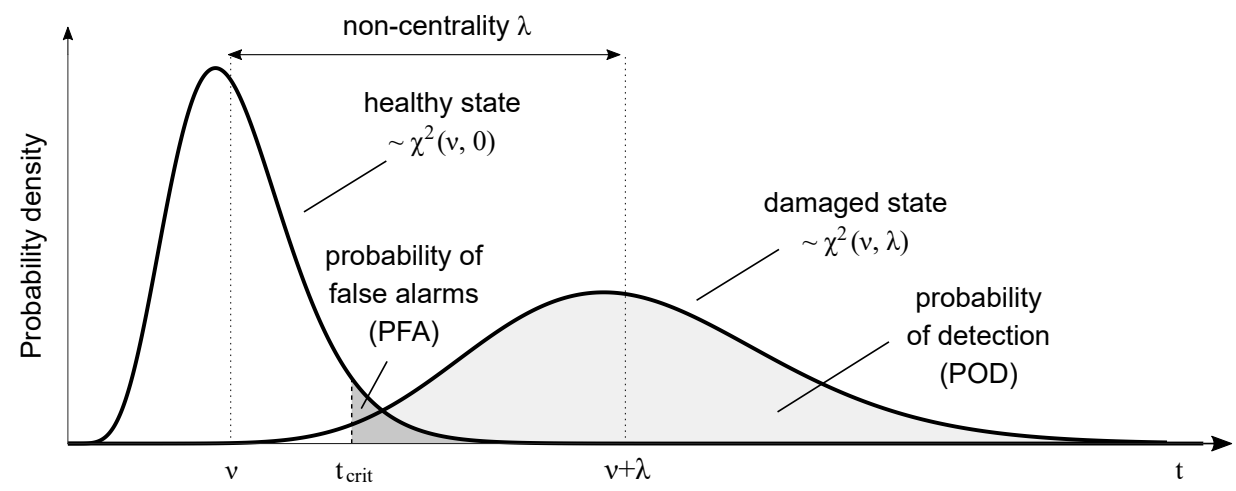

Figure 1: Reliability considerations based on the $\chi^{2}-$ distribution

which is a scalar test statistic that follows a $\chi^{2}$-distribution with $\nu$ degrees of freedom and the non-centrality $\lambda$, which is the shift in the mean value of the test response, yielding

$$
\nu=\operatorname{rank}\left(\mathcal{J}^{T} \boldsymbol{\Sigma}^{-1} \mathcal{J}\right)
$$

and

$$
\lambda=\boldsymbol{\delta}^{T}\left(\mathcal{J}^{T} \boldsymbol{\Sigma}^{-1} \mathcal{J}\right) \boldsymbol{\delta}
$$

The $\chi^{2}$-distributions of the test statistic in the reference and damaged states are visualized in Fig. 1. In this figure, the probability density functions are shown for both states. In the reference state, the mean value is equal to $\nu$ and $\nu+\lambda$ in the damaged state, and the non-centrality is the difference between the mean values. Moreover, the figure clarifies that the distributions overlap and shows how the safety threshold value $t_{\text {crit }}$ is linked to the probability of false alarms in the reference state and to the probability of detection in the damaged state. The structure is considered damaged if the test statistic exceeds this threshold and an alarm is issued. More information on the calculation of the safety threshold, the non-centrality, and a physical interpretation of the number of degrees of freedom is given in Section 4.4.

\section{Method to Determine the Minimum Detectable Damage}

Before deriving the minimum detectable damage, it is necessary to introduce how damage can be modelled and how its effect on the distribution of the damage detection test can be evaluated through the Fisher information. For this purpose, structural design parameters are chosen for the monitoring parameter vector $\boldsymbol{\theta}$ and for the analysis of minimum detectable damages therein.

Using finite element (FE) theory, the structure can be discretized, meaning it can be divided into finite elements while assigning structural design parameters to each element, e.g. material properties and crosssectional values. Optionally, structural components can be defined by assigning the same design parameters 
to multiple elements. Based on the expected damage scenarios, a set of design parameters, denoted by $\theta_{h}$ with $h=1,2, \ldots, H$, is selected for monitoring and stored in vector $\boldsymbol{\theta} \in \mathbb{R}^{H}$, which focuses the damage diagnosis on local structural components. To avoid bias in the analysis of the minimum detectable damage, the design parameters should match the actual properties of the investigated structure, and hence, be based on a well-calibrated model. Once damage is parametrized through changes in design parameters in $\boldsymbol{\theta}$, they are linked to the residual vector $\boldsymbol{\varepsilon}$ from Eq. (6) through the sensitivity matrix $\mathcal{J}$, which can be computed analytically through a series of first-order sensitivity analyses. In the first step, the structural design parameters of the finite element model are linked to the numerical modal parameters. Secondly, the modal parameters are linked to the Gaussian residual, as explained in detail in the referenced literature, see $[19,21]$. The damage detection test from Eq. (10) includes this sensitivity, and thus, the information from the physical model. Moreover, it contains the Fisher information

$$
\mathbf{F}=\mathcal{J}^{T} \boldsymbol{\Sigma}^{-1} \mathcal{J}
$$

which is a measure for the information on the parameters that is contained in the damage-sensitive feature. The Fisher information considers both the residual's sensitivity towards changes in the parameters and the uncertainty related to the estimation of the residual. If the sensitivity matrix is evaluated with respect to structural design parameters, as explained above, each main diagonal value of $F_{h h}$ becomes a measure for the detectability of damage in the respective design parameter $\theta_{h}$.

\subsection{Predictive Formula for the Minimum Detectable Damage}

A formula for the minimum detectable damage in any structural design parameter $\theta_{h}$ is derived by combining the considerations regarding the change vector from Eq. (7), the non-centrality from Eq. (12), and the Fisher information from Eq. (13). For the derivation, the effect of a single parameter change $\Delta \theta_{h}=\left(\theta_{h}-\theta_{h}^{0}\right)$ is analyzed while assuming no change in all other parameters. For this case, all entries of the statistical change vector $\boldsymbol{\delta}$ from Eq. (7) are zero except from the $h$-th entry, which is

$$
\delta_{h}=\sqrt{N}\left(\theta_{h}-\theta_{h}^{0}\right)
$$

and the non-centrality of the damage detection test in Eq. (12) reduces to $\lambda_{h}=F_{h h} \delta_{h}^{2}$. A reliable test result is given if the structural change and thus $\delta_{h}$ is sufficiently large, because then, the test distribution in the damaged state is well-separated from the reference state as in Fig. 1, with the decisive parameter being the non-centrality. In other words, defining a minimum value $\lambda_{\min }$ for the non-centrality is equivalent to defining a minimum damage detectability. The minimum detectable change in each parameter satisfies

$$
\lambda_{h}=F_{h h} \delta_{h}^{2}=\lambda_{\min }
$$

Details on how to calculate the minimum non-centrality $\lambda_{\min }$ based on the allowable PFA and the desired POD will be given in the subsequent section. Solving Eq. (15) for $\delta_{h}$ and plugging it into Eq. (14) results 


$$
\theta_{h}-\theta_{h}^{0}=\sqrt{\frac{\lambda_{\min }}{N \cdot F_{h h}}} .
$$

To obtain a formula for the minimum detectable damage in percent of the design parameter, the expression is normalized by the reference parameter $\Delta_{h}=\left(\theta_{h}-\theta_{h}^{0}\right) / \theta_{h}^{0}$ and the data length is substituted by the product of the measurement duration and the sampling frequency $N=T f_{s}$. The resulting formula

$$
\Delta_{h}=\frac{1}{\theta_{h}^{0}} \sqrt{\frac{\lambda_{\min }}{T f_{s} \cdot F_{h h}}}[\%]
$$

allows for the minimum detectable damage to be determined, i.e. the minimum change in any structural design parameter $\theta_{h}$ that is necessary to achieve a reliable damage detection.

\subsection{Minimum Non-centrality $\lambda_{\min }$}

The non-centrality $\lambda$ quantifies the degree of separation between the $\chi^{2}$-distributions related to the reference and damaged state, so it can be interpreted as a reliability index. The larger the non-centrality, the clearer the damage diagnosis and the higher the detectability of damage. Defining a minimum detectable damage is, therefore, equivalent to requiring a minimum non-centrality $\lambda_{\min }$. In the following, a reliability concept is developed to determine an appropriate value for $\lambda_{\min }$, by tying it back to more intuitive measures such as the acceptable PFA and the desired POD.

The $\chi^{2}$-distribution. The test statistic $t$ is approximately $\chi^{2}(\nu, \lambda)$-distributed, where $\nu$ is the number of degrees of freedom and $\lambda$ the non-centrality. The number of degrees of freedom $\nu$ can be treated as a known constant, which depends on the complexity of the monitoring problem, see Eq. (11). More precisely, it depends on the number of monitoring parameters in relation to the modes of vibration used for damage screening, so it remains unchanged throughout the damage diagnosis. Next to the theoretical formula for $\nu$, it can also be estimated empirically by evaluating the test statistic for multiple data sets from the structure in the reference state and fitting a central $\chi^{2}$-distribution to the histogram. The second parameter, the non-centrality $\lambda$, is zero in the reference state and different from zero in the damaged state, see Eq. (12). With the non-centrality and the number of degrees of freedom at hand, the PDF of the test statistic $f_{\chi^{2}(\nu, \lambda)}$ is uniquely defined for any health state.

Probability of False Alarms (PFA). This value quantifies how often the test mistakenly diagnoses a reference structure as damaged. Based on the user-defined PFA, which is also known as the type I error, the safety threshold value $t_{\text {crit }}$ can be defined that discriminates the reference state from the damaged one, see Fig. 1. Its value is determined as the $(1-\mathrm{PFA})$ quantile of the reference state PDF, such that

$$
\mathrm{PFA}=1-\int_{0}^{t_{\mathrm{crit}}} f_{\chi^{2}(\nu, 0)}(t) d t .
$$

For civil engineering structures, the PFA could, for example, be set to $0.3 \%$ or $5 \%$, or lower, with the result that, on average, one out of 333 or 20 tests falsely diagnose a structure as damaged. 
Probability of Detection (POD). This value describes the probability that a damaged structure is correctly classified as damaged. It is the complement of the false-negative rate (also known as the type II error) and is defined as

$$
\mathrm{POD}=\int_{t_{\mathrm{crit}}}^{\infty} f_{\chi^{2}(\nu, \lambda)}(t) d t
$$

see also Fig. 1. Its desired minimum value should be set according to the anticipated damage consequences. For load-bearing components, for instance, it could be aligned with the prescribed reliability concepts for structural health monitoring. In Canada, the code-based reliability index for the assessment of highway bridges ranges between 2.5 and 4.0 [47], depending on the damage consequences on element level (sudden vs. gradual failure), system level (total collapse vs. local failure), and inspection level, which translates into a probability of detection that ranges from $\mathrm{POD}=99.379 \%$ to $\mathrm{POD}=99.997 \%$. The ISO norm requires a reliability index between 2.3 and 4.3 [48], equivalent to a POD of $98.928 \%$ and $99.999 \%$. All mentioned reliability indices are based on the ultimate limit state, and a reference period of one year (S6-19) and 50 years (ISO-13822). Since the minimum detectable damage is calculated for each design parameter $\theta_{h}$, see Eq. (17), a different reliability index could be assigned to each parameter.

Minimum Non-centrality. Ultimately, the minimum non-centrality $\lambda_{\min }$ can be determined based on the desired PFA and POD with Eq. (19). In this equation, the non-centrality is the only unknown parameter, as the degrees of freedom $\nu$ are known from the reference state, $t_{\text {crit }}$ is set based on the desired PFA and the POD is set by the user. Through numerical iteration, $\lambda$ is varied until the theoretical distribution for the damaged state is sufficiently shifted to the right and the desired POD is achieved in Eq. (19) for $\lambda=\lambda_{\min }$. Note that the minimum non-centrality increases with increasing $\nu$, because the reference state PDF becomes wider and the damaged state PDF has to be shifted further to the right to guarantee a sufficient degree
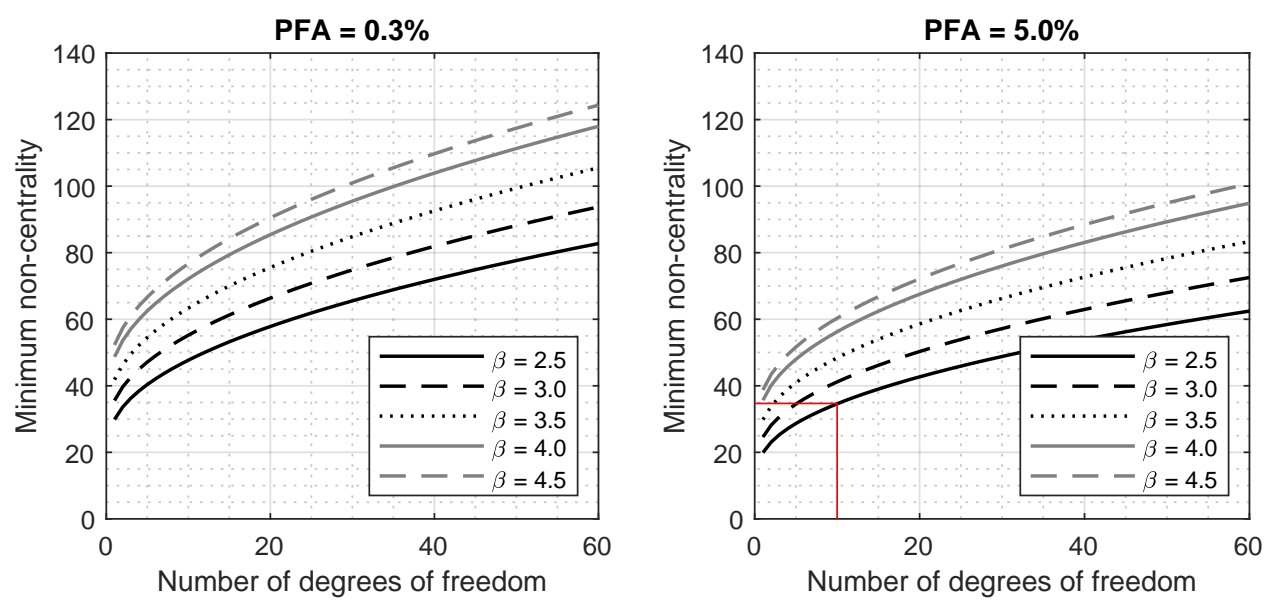

Figure 2: Minimum non-centrality for a probability of false alarms (PFA) of $0.3 \%$ and $5 \%$, and reliability indices $\beta$ of $2.5,3.0$, 3.5, 4.0, and 4.5 
of separation. The relation is visualized in Fig. 2, where the minimum non-centrality is plotted over the number of degrees of freedom for varying PFA and POD. It can be appreciated that, with an increasing number of monitoring parameters, the minimum non-centrality increases and the damage sensitivity for each monitoring parameter decreases, as per Eq. (17).

To conclude, the non-centrality $\lambda_{\min }$ is a problem-specific reliability index, which could be defined individually for each monitoring parameter. It can be derived from a user-defined PFA and POD and tied back to national safety standards.

\subsection{Interpretation}

The derived formula for the minimum detectable damage in Eq. (17) demonstrates that detectability depends on the information content of the damage-sensitive feature, properties related to the measurement environment and the reliability requirement towards the damage diagnosis, which can be tied back to the expectations from Section 2:

(a) Damage-sensitive feature and its sensitivity. The sensitivity of the feature towards changes in a structural design parameter $\theta_{h}$ is quantified through the sensitivity vector $\mathcal{J}_{h}$. The higher the sensitivity, the higher the main diagonal of the Fisher information $F_{h h}=\mathcal{J}_{h}^{T} \boldsymbol{\Sigma}^{-1} \mathcal{J}_{h}$, which, in turn, results in a smaller minimum detectable damage in $\theta_{h}$, cf. Eq. (17). The sensitivity itself depends on the damagesensitive feature, the number and location of sensors, the number of modes present in the data, and the parameter's contribution to the structural integrity.

(b) Signal-to-noise ratio. The uncertainties in the estimation of the damage-sensitive feature due to stochastic loads and noisy data are related to the signal-to-noise ratio of the measurements. Measurement records with strong structural signals and low noise components result in a high signal-to-noise ratio, and thus, low uncertainties. This is reflected through a low value for the covariance $\boldsymbol{\Sigma}$. The smaller the covariance, the higher the Fisher information $F_{h h}=\mathcal{J}_{h}^{T} \boldsymbol{\Sigma}^{-1} \mathcal{J}_{h}$, and the smaller the minimum detectable damage.

(c) Measurement duration. An increased measurement duration in the testing phase leads to a more reliable computation of the damage-sensitive feature with lower uncertainty regarding system parameters. Consequently, smaller changes can be detected at the same significance level, which is accounted for in Eq. (17). The minimum detectable damage appears to be inversely proportional to the square root of the measurement duration $T$. This also means that the damage detectability can be improved by increasing the measurement duration. In fact, the formula from Eq. (17) can be re-arranged and solved for the measurement duration

$$
T_{h}=\frac{1}{\left(\Delta \theta_{h}\right)^{2}} \frac{\lambda_{\min }}{f_{s} F_{h h}},
$$

in which case it can be used to determine the required measurement duration to reliably detect an anticipated parameter change $\Delta \theta_{h}=\left(\theta_{h}-\theta_{h}^{0}\right)$. 
(d) Reliability requirements for test results. Higher reliability requirements, i.e. a more conservative safety threshold for the reference state and a higher POD for the testing state, may be convenient for the operator of the SHM system but also increase the minimum non-centrality $\lambda_{\min }$. Consequently, higher damage magnitudes are required for successful detection, see Eq. (17), which reduces the overall detectability. Another factor that increases the minimum non-centrality is the number of monitoring parameters, see Fig. 2, so the number of monitoring parameters should be kept reasonably low. The number of monitoring parameters can be reduced by substructuring the model and assigning the same monitoring parameters to entire monitoring components instead of individual finite elements.

\subsection{Application to Data-driven Tests}

In the previous sections, the damage-sensitive feature was linked to structural design parameters in $\boldsymbol{\theta}$ to quantify the minimum detectable damage. In practical applications, other parameter sets can also be selected for monitoring (such as the modal parameters), or the damage detection test from Eq. (10) can be applied without any parametrization. The corresponding damage detection tests are data-driven [22, 30], because no information from $\mathrm{FE}$ models is required. In this section, the minimum detectable damage in the structural design parameters is analyzed for data-driven tests, and it is shown that the predictions from Eq. (17) remain valid.

When choosing modal parameters for monitoring, i.e. the poles and modes shapes from system (3), the monitoring parameters can be estimated from data in the reference state, and so can the corresponding sensitivity matrix [22]. For damage detection, the same statistical test is used (see Section 3), in combination with the monitoring parameter vector and sensitivity matrix

$$
\boldsymbol{\eta}=\left[\begin{array}{c}
\operatorname{diag}(\boldsymbol{\Lambda}) \\
\operatorname{vec}(\boldsymbol{\Psi})
\end{array}\right], \quad \mathcal{J}_{\text {mod }}=\left.\frac{\partial E_{\boldsymbol{\eta}}[\boldsymbol{\varepsilon}]}{\partial \boldsymbol{\eta}}\right|_{\boldsymbol{\eta}=\boldsymbol{\eta}^{0}} .
$$

Then, the mean of the Gaussian residual from Eq. (9) is $\mathcal{J}_{\text {mod }} \boldsymbol{\delta}_{\text {mod }}$, with $\boldsymbol{\delta}_{\text {mod }}=\sqrt{N}\left(\boldsymbol{\eta}-\boldsymbol{\eta}^{0}\right)$. Another data-driven test is the non-parametric test, where changes in the residual entries are detected without parametrizing it, so the sensitivity matrix simplifies to the identity matrix, $\mathcal{J}_{\text {dat }}=\mathbf{I}[22,30]$, and the mean value to $\boldsymbol{\delta}_{\mathrm{dat}}$, which is the mean of the Gaussian residual. To modify the test from Eq. (10) for the data-driven tests, the sensitivity matrix $\mathcal{J}$ is replaced by $\mathcal{J}_{\text {mod }}$ or $\mathcal{J}_{\text {dat }}$, respectively. Since the residual's mean is independent from the used parametrization, it holds

$$
\mathcal{J} \boldsymbol{\delta}=\mathcal{J}_{\text {mod }} \boldsymbol{\delta}_{\text {mod }}=\mathcal{J}_{\text {dat }} \boldsymbol{\delta}_{\text {dat }},
$$

and thus, the non-centrality $\lambda=\boldsymbol{\delta}^{T}\left(\mathcal{J}^{T} \boldsymbol{\Sigma}^{-1} \mathcal{J}\right) \boldsymbol{\delta}$ from Eq. (12) is independent of the parametrization. As a consequence, a structural damage that corresponds to a change in design parameter $\theta_{h}$ leads to the same non-centrality $\lambda$ of the test, regardless of its parametrization. That means that the prediction 
of the minimum detectable damage from Eq. (17) is also valid for data-driven tests, where either modal parameters or the residual entries are tested for damage without the information from a FE model. It should be emphasized that, in all cases, the prediction is in percent of a model-based design parameter. Nonetheless, the predicted minimum detectable damage differs depending on the applied tests, because the number of degrees of freedom $\nu$, and therefore, $\lambda_{\min }$ change with the used parametrization.

\subsection{Summary}

To conclude this section, a flowchart is given in Fig. 3 that summarizes the method for the prediction of the minimum detectable damage. The flowchart suggests defining the parameters of the test distribution based on theoretical formulas; however, the number of degrees of freedom $\nu$ and the safety threshold $t_{\text {crit }}$ can also be determined empirically based on a Monte Carlo experiment. In this case, the test statistic is repeatedly evaluated based on vibration data from the undamaged structure. Next, it is plotted in a histogram and a $\chi^{2}$-distribution is fitted with $\nu$ being the only variable. The empirical curve fitting is an appropriate means to validate the theoretical distribution properties and to verify the input parameter choice for practical applications. Detailed explanations on the application and the validation of the method follow in the next section.

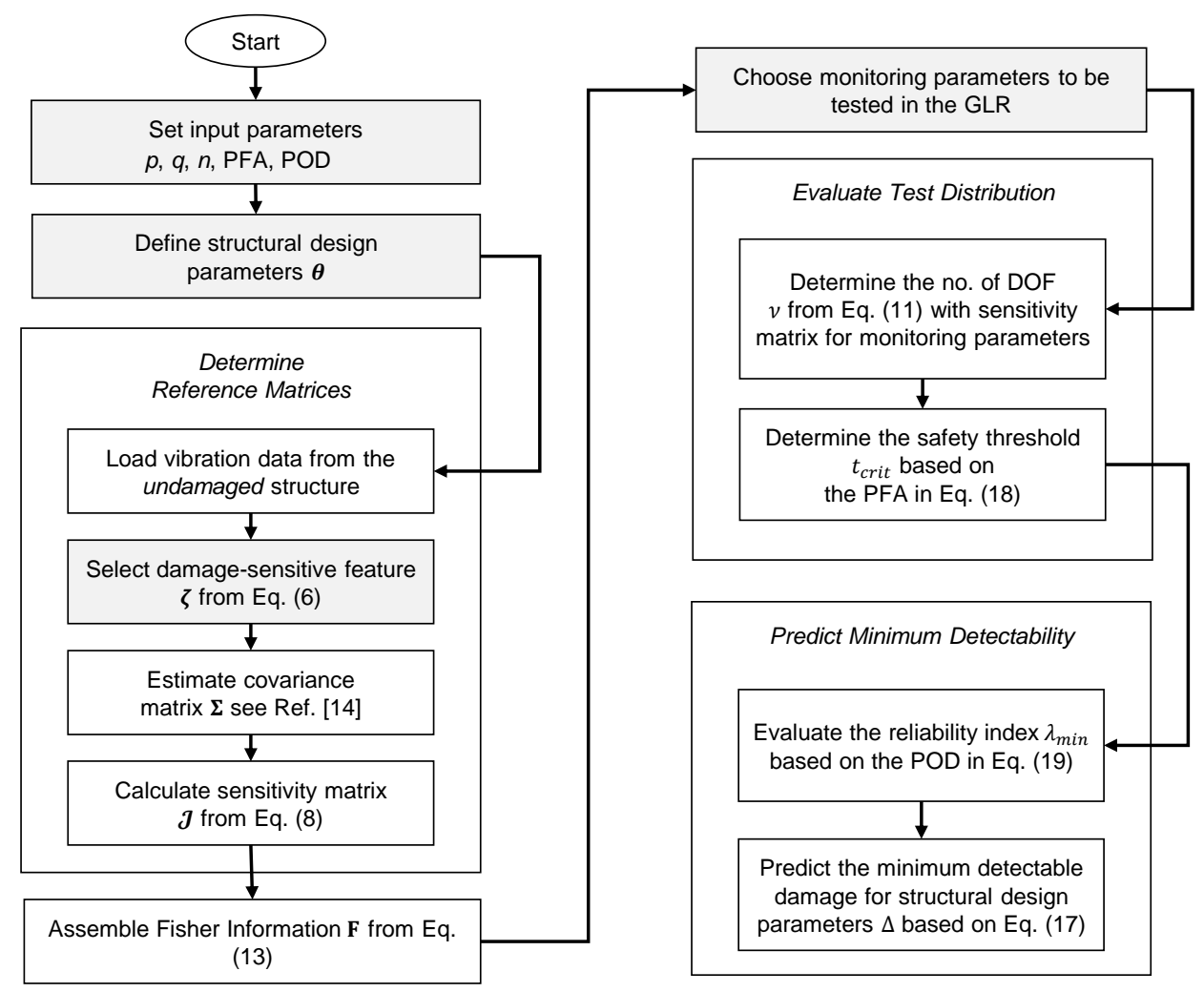

Figure 3: Flowchart of the proposed method 


\section{Application to a Pin-Supported HSS Beam}

In this section, the developed method is applied to several data sets of both simulated and experimental data from a pin-supported beam. In Monte Carlo studies, the empirical distributions of the damage detection tests in the reference and damaged states are evaluated to validate the predicted minimum detectable damages.

\subsection{Structure, Instrumentation and Parametrization}

The examined structure is a $4.11 \mathrm{~m}$-long laboratory beam on bolted pin supports, see Fig. 4 . It has a hollow structural steel cross-section, HSS $152 \times 51 \times 4.78 \mathrm{~mm}$, with a modulus of elasticity of $E=200,000$ $\mathrm{MPa}$ and a total mass of $m=56.8 \mathrm{~kg}$. For excitation, an electrodynamic shaker with a moving mass of $360 \mathrm{~g}$ is placed on the structure injecting a white noise signal vertically into the beam. Moreover, eight wireless seismic sensors (Tromino Tromographs) are placed at sensor locations P1-P8 and synchronized through radio communication. The sensor choice allows for different data quantities to be analyzed, as each sensor module includes tri-axial accelerometers, high-gain velocimeters, and low-gain velocimeters. However, for the damage detection studies presented in this paper, only three vertical acceleration channels at P1, P4, and P7 are used (except from Section 5.4.3 where different sensor configurations are analyzed). The signals are originally sampled at $512 \mathrm{~Hz}$ and later downsampled to $330 \mathrm{~Hz}$. An additional high-pass filter is applied to suppress non-structural signal components below $4 \mathrm{~Hz}$.

For parametrization, a 3-D FE model with 104 degrees of freedom is created in Ansys and discretized into 18 finite beam elements with a length of $22.8 \mathrm{~cm}$ each. Furthermore, nine components are defined by
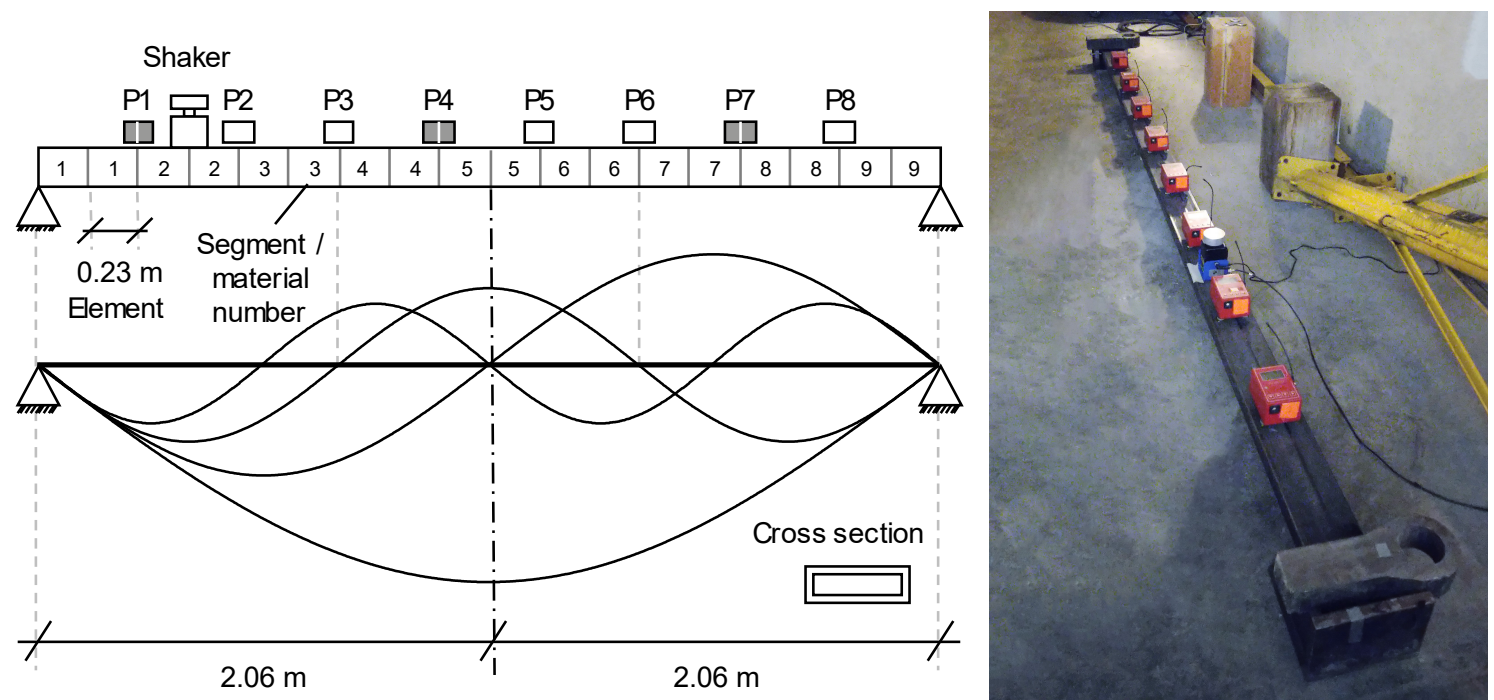

Figure 4: Pin supported HSS beam. Schematic depiction of the shaker position, the sensor positions P1, P4, and P7, as well as the analytical modes shapes of the first four modes of vibration (left), and a photo of the experimental test setup (right) 
assigning the same material constants to two consecutive FE, see Fig. 4. Each material is characterized through its modulus of elasticity and mass value, so in total, the structural design parameters include nine mass parameters and nine stiffness parameters. Damage is, therefore, defined as a change in the modulus of elasticity or the mass value of any of the eight beam segments. To account for the additional mass of the shaker, which has a total mass of $3.6 \mathrm{~kg}$, as well as the mass of each sensor module $(1.28 \mathrm{~kg})$, point masses are defined at the corresponding nodes of the FE model. The first study is based on simulated data, meaning the model was not only used to parametrize damage but also to generate acceleration records for both the reference and the damaged structure. For those records, the same input is applied as for the experiments and uniformly distributed noise was added to the generated outputs (with a magnitude corresponding to $5 \%$ of the output variance) to mimic realistic test conditions.

Within the considered frequency band, the first four vertical modes of vibration are present, which are used to screen the beam for damages. Their schematic mode shapes and natural frequencies are summarized in Fig. 4 and Table 1, respectively. The data-driven modal parameters are estimated based on stochastic subspace-based system identification to verify the quality of the FE model, i.e. to verify that the natural frequencies and mode shapes estimated from data are consistent with the model-based ones for both the reference state and the damaged state. An in-depth model-updating was deemed unnecessary. However, the modal parameter estimates are not required for damage diagnosis, as the numerical modal parameters are used instead. For consistency across all damage diagnosis studies, the time lags for the block Hankel matrix estimate are set to $p+1=q=13$ with a system order of $n=16$. Ultimately, the reliability requirements regarding the test results are defined through a maximum PFA of 5\% and a minimum POD of 99.4\% $(\beta=$ $2.5)$.

\begin{tabular}{ccrrr}
\hline \multicolumn{5}{c}{ Natural Frequencies [Hz] } \\
\hline \multirow{3}{*}{ FEA } & \multicolumn{3}{c}{ OMA } \\
\cline { 3 - 5 } No. & Ref. & Ref. & $\Delta_{5}=5 \%$ & $\Delta_{8}=5 \%$ \\
\hline 1. & 8.75 & 8.75 & $8.79(+0.43 \%)$ & $8.83(+0.93 \%)$ \\
2. & 34.4 & 35.7 & $35.7(-0.00 \%)$ & $35.6(-0.29 \%)$ \\
3. & 79.6 & 79.2 & $78.9(-0.34 \%)$ & $78.8(-0.53 \%)$ \\
4. & 141.4 & 142.5 & $142.3(-0.11 \%)$ & $142.5(-0.66 \%)$ \\
\hline
\end{tabular}

Table 1: Natural frequencies from finite element analysis (FEA) and operational modal analysis (OMA) for the reference state and a $5 \%$ mass change in Segment 5 and 8 


\subsection{Diagnosis Validation Procedure (Monte Carlo)}

The primary goal of this study is to validate the predictive formula for the minimum detectable damages. This is done by verifying the theoretical properties of the $\chi^{2}$-distribution of the test for both the reference and the damaged state. The damage detection test from Eq. (10) yields a scalar value for each run, and its distribution is evaluated in a Monte Carlo experiment, meaning the damage diagnosis is repeated 100 times (or more), before and after a damage event. The empirical distributions can be then described through histograms. The validation procedure is illustrated in Fig. 5. First, the test is repeatedly applied to the structure while plotting the test results in a control chart, see Fig. 5 (left). The control chart indicates that damage occurred during monitoring because, from a certain point onward, almost all test statistics exceed the safety threshold value. Dividing the data into the undamaged and the damaged segment and plotting the empirical distributions in a histogram, as it is done in Fig. 5 (right), allows for the statistical properties of the empirical distributions to be examined and compared to the theoretical ones from Fig. 1.

The main idea is to calculate the minimum detectable damage for a fixed measurement duration, to set the damage to the minimum detectable damage, and to compare the change in the statistical distribution of the test to the theoretical change. If the predictions are correct, the resulting non-centrality, i.e. the shift in the mean value of the empirical distribution, is identical to the minimum non-centrality $\lambda_{\min }$ defined through the reliability concept. This state can also be interpreted visually, as the empirical histograms fill the area under the theoretical distributions from Fig. 1. In addition to the visual comparison, the empirical power of detection is evaluated. The empirical POD is identical to the theoretical one from Eq. (19) if the prediction of the minimum detectable damage is accurate, and usually close to $100 \%$, see Fig. 1.

$$
\mathrm{POD}_{e m p}=\operatorname{Pr}\left[t>t_{\text {crit }}\right] \stackrel{!}{=} 99.4 \%
$$

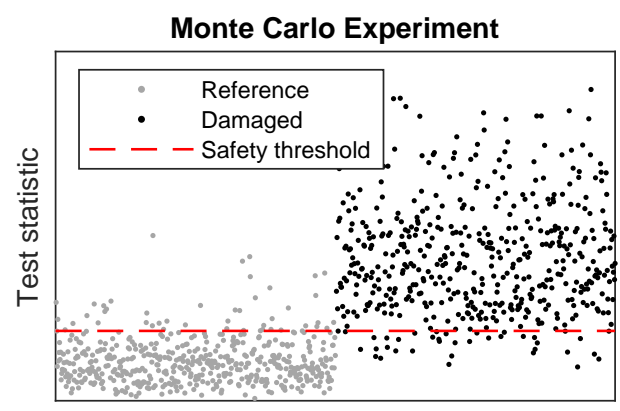

Time sample

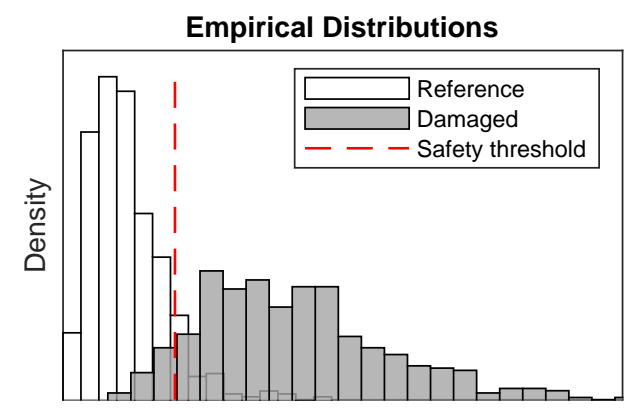

Test statistic

Figure 5: Monte Carlo experiment (left) and histograms of empirical $\chi^{2}$-distribution before and after a damage event (right side) 


\subsection{Numerical Validation}

The first study is based on simulated vibration data and focuses on the minimum detectable change in stiffness, i.e. a change in the modulus of elasticity in any of the nine materials or beam segments, where the structural design parameter vector is defined as

$$
\boldsymbol{\theta}=\left[\begin{array}{lll}
E_{1} & \cdots & E_{9}
\end{array}\right]^{T} .
$$

\subsubsection{Monitoring Model-based Design Parameters}

The following paragraphs explain the validation procedure for the minimum detectable damages when monitoring the model-based design parameters. The procedure is divided into four states, i.e. (i) the reference state, (ii) the training state, (iii) the prediction of the minimum detectable damages, and (iv) the testing state.

Reference State. In the reference state, one long data set is generated for the reference structure to compute the required quantities for the damage detection test, that is, the null space $\mathbf{S}_{0}$, the residual's covariance $\boldsymbol{\Sigma}$, the sensitivity matrix $\mathcal{J}$ from Eq. (8), and the corresponding Fisher information $\mathbf{F}$ from Eq. (13). The Fisher information is also shown in Fig. 7. The main diagonal values $F_{h h}$ of the square matrix are highlighted in grey, as they are used in the following steps as a measure for the detectability of damage.

Training State. During training, the safety threshold value $t_{\text {crit }}$ of the test statistic and the number of degrees of freedom $\nu$ of its distribution are evaluated based on data from the reference structure. First, a histogram of the test statistic is created by applying the damage detection test from Eq. (10) to 200 data sets of $30 \mathrm{~s}$ length. Secondly, the number of degrees of freedom $\nu$ is verified by fitting the central $\chi^{2}-$ distribution to the histogram, and ultimately, the safety threshold value $t_{\text {crit }}$ is set based on the acceptable PFA, see Eq. (18). The results for the HSS beam are visualized in Fig. 6. The empirical distribution from the Monte Carlo simulation (white histogram) is nearly identical to the theoretical distribution (dotted black line).

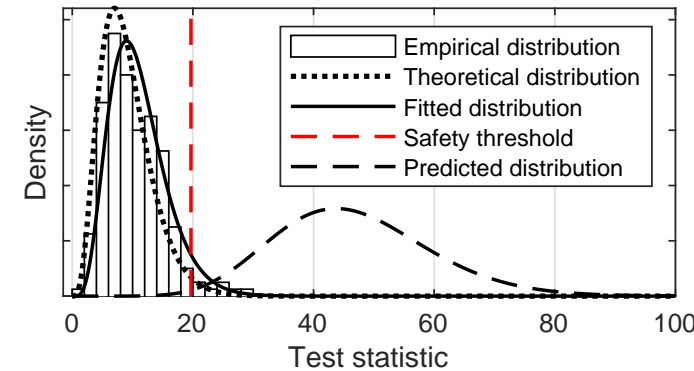

Figure 6: Empirical $\chi^{2}$-distribution (training state)

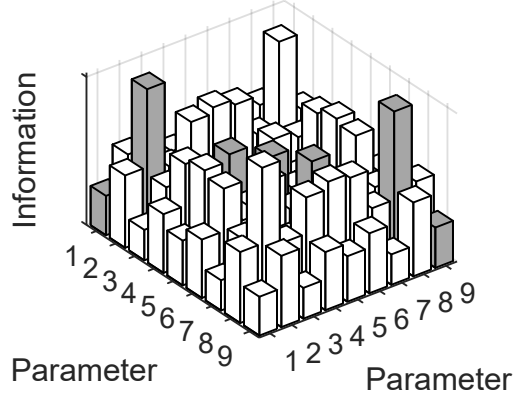

Figure 7: Fisher information (reference state) 
Fitting a $\chi^{2}$-distribution to the histogram yields $\nu=10$ (solid black line), which is close to the theoretical value of $\nu=9$ from Eq. (11). The safety threshold that corresponds to a PFA of $5 \%$ is about 20 .

Prediction. To predict the minimum detectable stiffness change in each monitoring parameter for a measurement duration of $T=30 \mathrm{~s}$, one more input parameter is required, that is, the minimum noncentrality $\lambda_{\min }$. Since the minimum non-centrality not only depends on the PFA and POD, but also the number of degrees of freedom $\nu$, the prediction can be made after the training is completed. The PFA of $5 \%$, the POD of $99.4 \%$, and the number of degrees of freedom of $\nu=10$ translate into a non-centrality of $\lambda_{\min }=34.7$, see the red line in Fig. 2. With all input parameters at hand, the minimum detectable stiffness change can be calculated according to Eq. (17) and is presented in Table 2. This table shows that the SHM system is less sensitive to stiffness changes in beam segments $E_{1}, E_{3}, E_{7}$, and $E_{9}$.

\begin{tabular}{lccccccccc}
\hline \multicolumn{10}{c}{ Minimum } \\
\hline Metectable Damage [\%] \\
\hline Parameter change & 8.7 & 4.4 & 9.5 & 5.6 & 5.6 & 5.6 & 9.5 & 4.4 & 8.6 \\
\hline
\end{tabular}

Table 2: Minimum detectable change in the Young's modulus $E$ for each beam segment

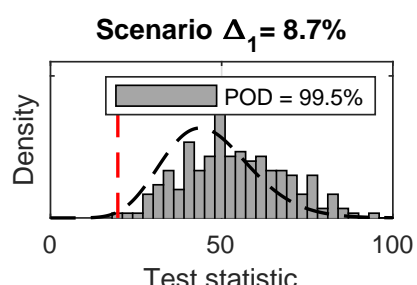

Test statistic

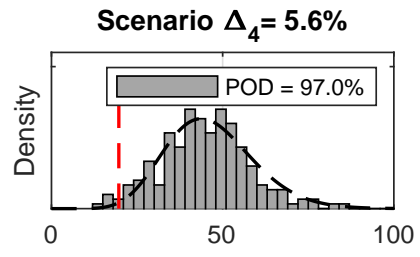

Test statistic

Scenario $\Delta_{7}=9.5 \%$

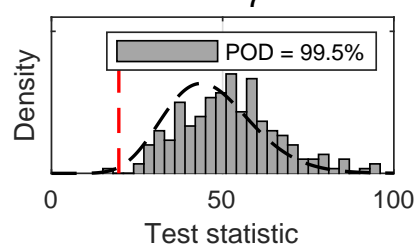

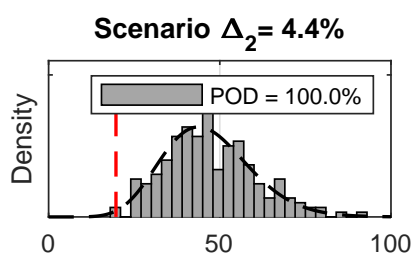

Test statistic

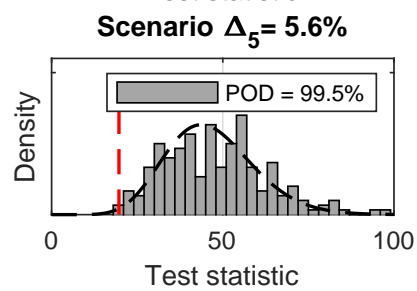

Scenario $\Delta_{8}=4.4 \%$

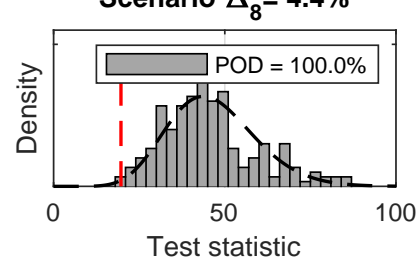

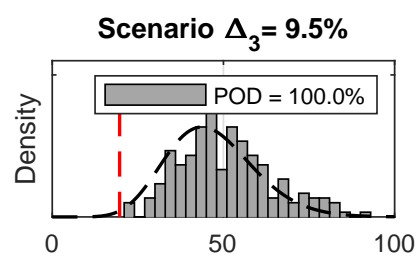

Test statistic

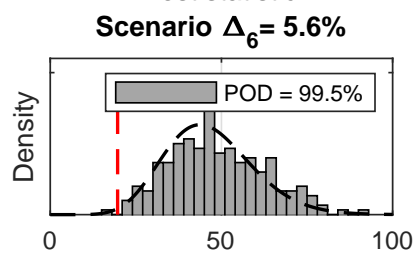

Test statistic

Scenario $\Delta_{9}=8.6 \%$

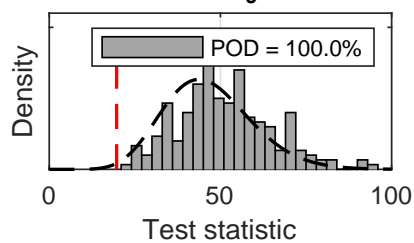

Figure 8: Empirical $\chi^{2}$-distribution (testing state) for all anticipated damage scenarios 
Testing State. During testing, vibration data from the damaged structure is examined. The main idea is to set the stiffness change to the minimum detectable damage from Table 2 and to verify whether the empirical distribution in the damaged state follows the theoretical one from the prediction. Since nine design parameters are defined, nine Monte Carlo simulations are performed-one for each damage scenario. All nine empirical distributions are shown in Fig. 8 together with the safety threshold and the empirical POD in the legends. The titles of the subfigures moreover repeat the minimum detectable damage in percent of the Young's modulus E, which is also tabulated in Table 2. For damage Scenario 2, for example, where the stiffness of the beam segment with the lowest minimum detectable damage is decreased by $\Delta_{2}=4.4 \%$, the empirical distribution from the Monte Carlo simulation (grey histogram) appears similar to the predicted one. The POD of $100.0 \%$ is also close to the theoretical one of $99.4 \%$, see Eq. (22). The POD ranges between $97.0 \%$ and $100 \%$ for all damage scenarios with a mean value of $99.4 \%$, so the validation of the predictive formula is successful.

\subsubsection{Monitoring with Data-driven Tests}

Next, the prediction of the minimum detectable damage is validated while monitoring modal parameters. Monitoring modal parameters means that the sensitivity matrix $\mathcal{J}$ in the damage detection test from Eq. (10) is exchanged with $\mathcal{J}_{\text {mod }}$, which is the sensitivity of the residual towards modal parameters, see Eq. (21). The approach is identical to the validation procedure for monitoring design parameters in the previous section. However, the modification of the test changes the minimum detectable damage, because the non-centrality $\lambda_{\text {min }}$ depends on the number of monitoring parameters, or more precisely, the number of degrees of freedom $\nu$ of the test in Eq. (11). The reference state remains unchanged and the Fisher information $\mathbf{F}$ in Eq. (13) is again evaluated with respect to the model-based design parameters $\boldsymbol{\theta}$ because damage is defined as a change therein. The FE model is, therefore, only used for the prediction of the minimum detectable damage, before the SHM system is installed, but not during monitoring. For conciseness, the validation procedure is only shown for damage in beam Segment 2, where the histograms for both the reference state and the damaged state with $\Delta_{2}=5.1 \%$ are plotted in one plot and juxtaposed to the validation chart of the model-based test in Fig. 9. The theoretical number of monitoring parameters increases from nine material constants to $2 m r=24$ parameters, which is close to the empirical number of degrees of freedom of the $\chi^{2}$-distribution of $\nu=25$. An empirical POD of $99.5 \%$ confirms that the predictions are accurate and validates the theoretical formulas for monitoring modal parameters.

Ultimately, the minimum detectable damages are evaluated for the non-parametric test. That means that the Gaussian residual is monitored directly and without parametrizing it, so the sensitivity matrix in the test from Eq. (10) is set to unity $\mathcal{J}_{\text {dat }}=\mathbf{I}$, and the test simplifies to $t=\boldsymbol{\zeta}^{T} \boldsymbol{\Sigma}^{-1} \boldsymbol{\zeta}$. The results for Segment 2 are visualized in Fig. 9, where the minimum detectable damage is now 7.6\%. Interestingly, the number of degrees of freedom of the fitted reference state distribution is $\nu=237$, and thus, considerably 


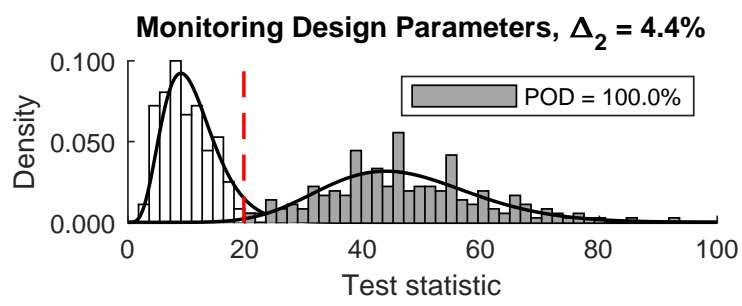

Monitoring Modal Parameters, $\Delta_{2}=5.1 \%$

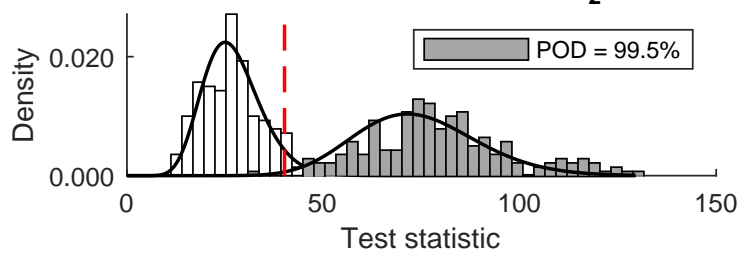

Monitoring the Residual Entries, $\Delta_{2}=7.6 \%$

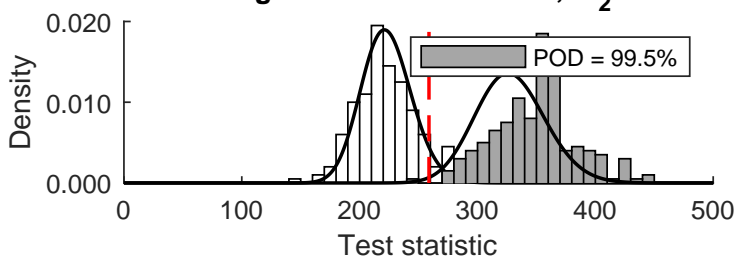

Figure 9: Empirical $\chi^{2}$-distribution (training and testing states) while monitoring stiffness changes

lower than the theoretical value of $\nu=\operatorname{rank}\left(\boldsymbol{\Sigma}^{-1}\right)=897$, which is the numerical rank of the covariance matrix from Eq. (11). This discrepancy may be due to a gradual decrease of its singular values with no clear jump, while the matrix is typically rank-deficient [49]. Here the advantages of the empirical approximation of $\nu$ become apparent, since the minimum detectable stiffness change can be predicted correctly with the empirical $\nu$, as validated by an empirical POD of $99.5 \%$, see Fig. 9 .

To sum up, the predictions of the minimum detectable damage are correct for both model-based and data-driven tests. For the non-parametric test, empirical curve fitting is necessary in the training state because the rank calculation yields a misleading value for the number of degrees of freedom. The number of degrees of freedom increases for the three examined tests from 10 over 25 to 237 . Correspondingly, the minimum non-centrality increases (as indicated in Fig. 2) and so does the minimum detectable stiffness change from $4.4 \%$ over $5.1 \%$ to $7.6 \%$, see Fig. 9. In other words, a low number of monitoring parameters leads to a high damage sensitivity. This concludes the validation of the theoretical investigations and paves the way for an experimental application with real vibration data in the subsequent section.

\subsection{Experimental Application}

The second study is based on real vibration data from the same structure and, to demonstrate the full capabilities of the developed framework, it focuses on the minimum detectable change in mass, where the 


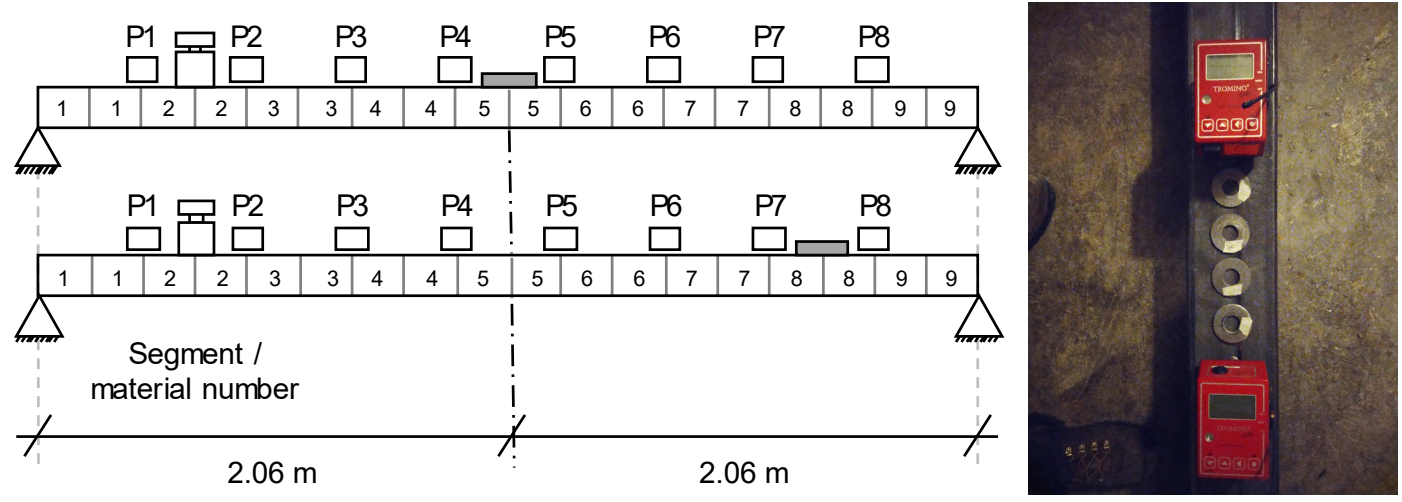

Figure 10: Arrangement of extra masses during laboratory tests. Schematic arrangement (left) and photo of extra masses (right)

structural design parameter vector is defined as

$$
\boldsymbol{\theta}=\left[\begin{array}{lll}
m_{1} & \ldots & m_{9}
\end{array}\right]^{T}
$$

To simplify the experimental procedure, the mass of one beam segment is increased by $5 \%$ by applying a loose extra mass of $0.31 \mathrm{~kg}$ on top of it. Subsequently, the minimum measurement duration is adjusted according to Eq. (20), so the applied mass change in the experiment corresponds to the minimum detectable mass change in the examined beam segment. The validation procedure for the minimum detectable damage is then identical to the one described in Section 5.3.1. For the evaluation of the empirical distributions in the Monte Carlo set up, the available 110 min-long record (2,178,000 samples) for each state is divided into data sets depending on the respective measurement duration.

\subsubsection{Extra Mass Near the Support}

First, an extra mass of $5 \%$ is applied to beam Segment 8, see Fig. 10. The validation for the modelbased and data-driven tests is summarized on the left side of Fig. 11. For the model-based test, the empirical distribution of the test in the reference state is close to the theoretical distribution, and a $5 \%$ mass increase can be detected with a POD of $96.6 \%$. So, the estimation of the minimum measurement duration, and thus, the minimum detectable damage is accurate. For the data-driven test, where the residual is parametrized with respect to modal parameters, the reference state is shifted towards higher values but the prediction is still correct with a POD of $98.1 \%$. The non-parametric data-driven test showed to be less robust than the parametric tests regarding its theoretical properties, as the reference state distribution is further shifted towards higher values. Additional studies showed some dependence between the empirical $\nu$ and the measurement duration. Nonetheless, when the measurement duration was determined in an iterative manner until the predicted minimum detectable damage in Segment 8 was $\Delta_{8}=5 \%$, as applied in the 

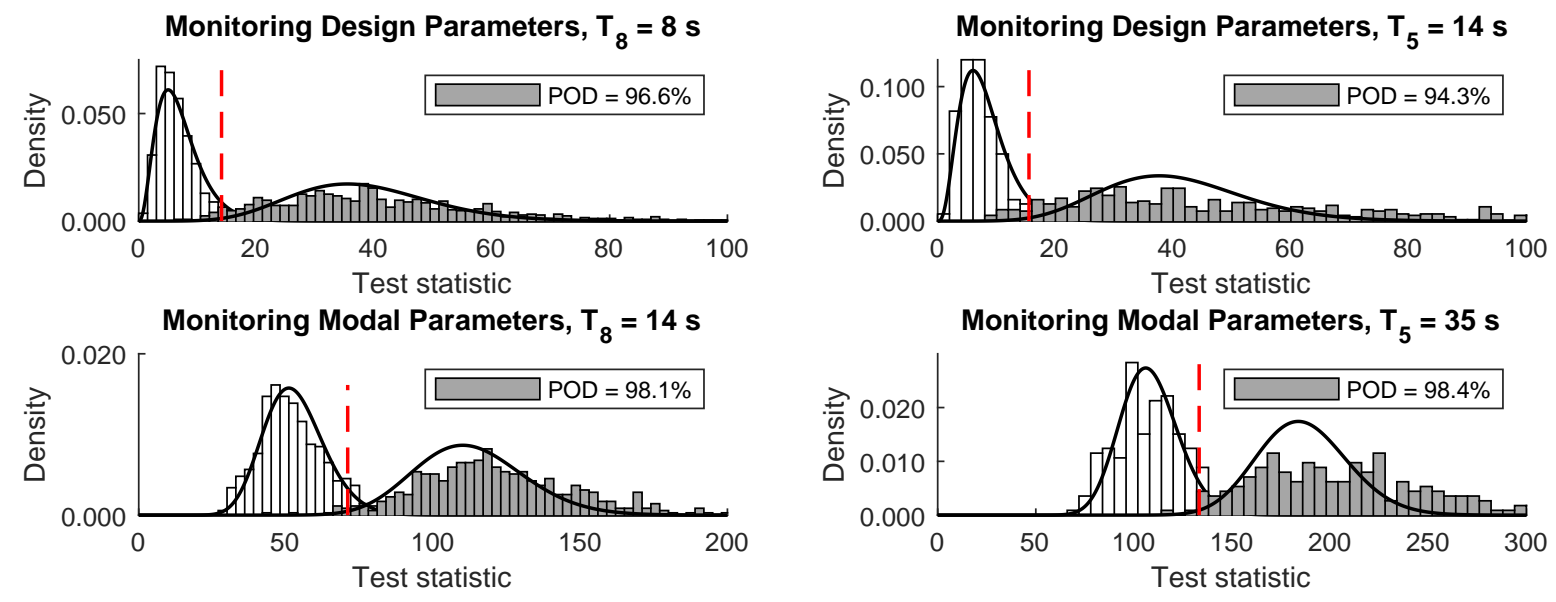

Monitoring Modal Parameters, $\mathrm{T}_{5}=35 \mathrm{~s}$
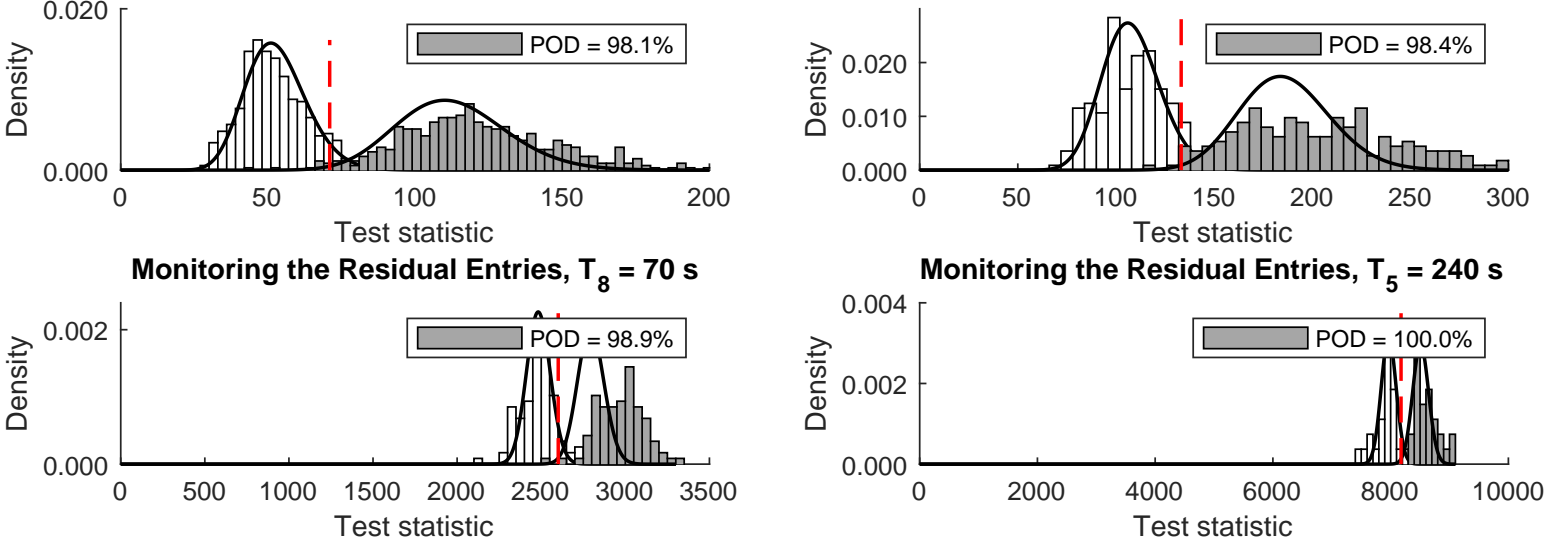

Figure 11: Empirical $\chi^{2}$ distribution from Monte Carlo experiment (training and testing state) while monitoring mass changes. Results for an $5 \%$ extra mass on Segment 8 (left) and on Segment 5 (right)

experiment, the prediction is accurate with a POD of $98.9 \%$.

For all three tests, the number of degrees of freedom increases from 7 over 53 to 2,484 and the minimum measurement duration that is necessary to detect a $5 \%$ mass change increases, respectively, from $8 \mathrm{~s}$ over $14 \mathrm{~s}$ to $70 \mathrm{~s}$. In all cases, the predictions could be validated with experimental data. Moreover, it has been shown that the measurement duration can be interpreted as a measure for the detectability of damage. In this sense, the model-based test is the most sensitive to small and local damages. It is also the most robust as the empirical distribution is identical to the theoretical one, regardless of the measurement duration.

\subsubsection{Extra Mass at Midspan}

Secondly, an extra mass of $5 \%$ is applied at midspan, i.e. beam Segment 5 from Fig. 10. The predictions could, again, be verified with a high POD of $94.3 \%, 98.4 \%$, and $100 \%$ for the model-based test and the datadriven tests, see Fig. 11 (right). The number of degrees of freedom increase from 8 over 108 to 7,968 and the required measurement duration increases from $14 \mathrm{~s}$ over $35 \mathrm{~s}$ to $240 \mathrm{~s}$. Overall, the required measurement duration to detect a $5 \%$ mass increase at Segment 5 is higher than it was for Segment 8. Correspondingly, the main diagonal entry of the Fisher information for Segment 5 is lower than for Segment 8, cf. $F_{55}$ and 
$F_{88}$ in Fig. 7.

\subsubsection{Varying Number and Location of Sensors}

To illustrate the robustness of the predictions, the validation is repeated for a varying number of sensors and sensor positions. Originally, the experiment was performed with eight equidistant sensors, as indicated in Fig. 10, so different subsets can be selected for the analysis. The prediction was accurate for one to eight sensors, which also means that stochastic subspace-based damage detection is possible using a single sensor. For conciseness, the results are not visualized here, as they are qualitatively identical to previous validation runs, summarized in Fig. 11.

\section{Discussion}

The results from the previous section show that the formula for the minimum detectable damage is accurate for simulated and real data, regardless of whether damage is implemented as a change in structural stiffness or mass. The predictions are made based on measurement data from the reference state and sensitivity vectors that link the damage-sensitive feature to selected structural design parameters from a FE model. Vibration data from the damaged state is only used for validation purposes. Therefore, the predictive formula is a useful tool to assess the performance of a damage detection method in conjunction with the chosen sensor layout and data acquisition setup, before damage occurs. Based on the theoretical and practical investigations, the following remarks can be made.

\subsection{Assessing the Damage Sensitivity}

Global monitoring approaches based on the natural system response to unknown excitation are often criticized because of their low sensitivity to small and local damages. The applied damage detection method, the SSDD, is a global approach but it appears to be highly sensitive to small structural changes in either mass and stiffness. For experimental data, a 5\% mass change could be detected reliably in one beam segment which, in relation to the entire beam mass, corresponds to a $0.6 \%$ mass change. For comparison, the results from classical frequency monitoring are summarized in Table 1 . The extra mass causes the natural frequencies of the first four modes of vibration to change by a maximum of $0.93 \%$. This frequency change is small and in the wrong direction, but nonetheless, a long measurement duration is required for an accurate estimation of the modal parameters. A common rule of thumb for practicing engineers is to set the measurement duration to $T=1000 \cdot T_{1}=110 \mathrm{~s}$, where $T_{1}$ is the natural period of the first mode of vibration [50]. In contrast, the measurement duration for the detection of the $5 \%$ mass change with the SSDD method was as low as $T=8 \mathrm{~s}$. The developed method allows a rigorous evaluation of the damage sensitivity for damage detection. 


\subsection{Significance of Parametrization}

For physical interpretation, changes in the damage-sensitive feature are parametrized, meaning they are linked to a structure-specific parameter vector through sensitivity matrices. For the determination of the minimum detectable damage, a parametrization with respect to structural design parameters in $\boldsymbol{\theta}$ from a FE model is inevitable, where each damage scenario is defined mathematically through a change in one of the parameters. For monitoring, a second parameter vector is defined, and the sensitivity of the damagesensitive feature towards these monitoring parameters is considered in the statistical test from Eq. (10). One approach is to define the same parameter vector for monitoring that was used to predict the minimum detectable damage, i.e. $\boldsymbol{\theta}$, in which case the structural parameters are tested for damage. Alternatively, other appropriate monitoring parameter vectors can be tested for damage, such as modal parameters by means of the parametrization $\boldsymbol{\eta}$, or ultimately, the entries of the Gaussian residual can be tested directly without any parametrization. This widens the field of monitoring parameters and enables the analysis of the minimum detectable damage for data-driven tests, as in $[22,30]$.

Since damage is defined as a change in a model-based design parameter, so in $\boldsymbol{\theta}$, each damage scenario has to be parametrized appropriately for the analysis of its minimum detectable damage, and the reference values of the design parameters have to match the structure for a correct analysis. For example, for the considered HSS beam, the axial stiffness $E A$ or bending stiffness $E I$ could have been chosen. Likewise, the cross-sectional area $A$ or the moment of inertia $I$, or underlying quantities such as the E-modulus, the beam height or width, or the mass density are possible monitoring parameters. Which parameter is the most appropriate depends on the anticipated damage scenarios and the stress distribution within the structure. In the case of the HSS beam, an appropriate damage parametrization demands that the extra masses are distributed along the beam segment, as damage was modelled as a change in the uniformly distributed mass (see Fig. 10).

The parametrization of the residual for monitoring is optional as it can also be evaluated directly. One desired effect that parametrization has is that it increases the damage sensitivity because the number of parameters can be reduced, which, in turn, leads to a lower minimum non-centrality. In this sense, the sensitivity matrix can be understood as a projection matrix that projects the residual onto a lowerdimensional space. Among the considered parametrizations for the HSS beam, the model-based test with the parametrization $\boldsymbol{\theta}$ has shown to be the most sensitive and robust. For data-driven monitoring, it is recommended to choose the parametrization with respect to modal parameters in $\boldsymbol{\eta}$ over the non-parametric test for robustness, whenever possible. The most compelling reason to use the model-based parametrization is, however, that the monitoring can be focused on user-defined parameters that are critical for the safety or serviceability of the structure. 


\subsection{Non-destructive Validation Methods}

The damage sensitivity has been evaluated with respect to stiffness and mass changes through the proposed approach. In most practical applications, it cannot be justified to modify the stiffness of structural components of safety-critical structures only to validate the effectiveness of a monitoring system. Having said that, the application of extra masses has shown to be a promising non-destructive validation method to demonstrate that the damage detection algorithm has been implemented and trained correctly for cases where damaging the structure is not an option.

\section{Conclusion}

This paper presents an approach to determine the minimum detectable damage for vibration-based structural health monitoring. All considerations regarding the damage sensitivity lead to a concise formula, see Eq. (17), that relates the minimum detectable change in percent of a model-based design parameter to the measurement duration during testing, as well as to a reliability index that incorporates the allowable probability of false alarms and probability of detection, and ultimately, the Fisher information which quantifies the sensitivity of the employed damage-sensitive feature and its uncertainty due to unknown excitation and noise effects. A finite element model and vibration data from the reference structure are required to determine the minimum detectable damage; however, the predictions are also valid for purely data-driven tests where no finite element model is used. The predictive formula is derived for the stochastic subspace-based residual but can be applied to any vibration-based damage-sensitive feature, provided its sensitivities and statistical properties can be characterized in the considered Gaussian framework. To illustrate the developments, the minimum detectable mass change in a steel beam is predicted and validated through a laboratory experiment. The application of extra masses appears to be a promising non-invasive validation technique to verify whether the algorithm is implemented correctly for applications where damaging the structure is not an option.

The predictive formula is a practice-friendly tool to utilize ambient vibrations from the reference structure for health monitoring and risk analysis. It relates changes in damage-sensitive features to a degradation of engineering design parameters that are decisive for the structural health and safety. This way, the performance of the considered damage detection method can be assessed before damage occurs, which is a relevant asset before deploying SHM in practice. Ultimately, it links SHM approaches to reliability analysis and helps to evaluate the value of monitoring information in general.

\section{Acknowledgments}

This work was supported by a strategic research grant from the Natural Sciences and Engineering Research Council of Canada, the German Academic Exchange Service, and a Mitacs Globalink Research 
Award.

\section{References}

[1] C. R. Farrar, K. Worden, An introduction to structural health monitoring, Philosophical transactions. Series A, Mathematical, physical, and engineering sciences 365 (1851) (2007) 303-315.

[2] C. Farrar, K. Worden, Structural health monitoring: A machine learning perspective, Wiley, Oxford, U.K., 2012.

[3] C. Boller, F.-K. Chang, Y. Fujino, Encyclopedia of structural health monitoring, John Wiley, Chichester West Sussex U.K., 2009.

[4] F. Moreu, X. Li, S. Li, D. Zhang, Technical specifications of structural health monitoring for highway bridges: New Chinese structural health monitoring code, Frontiers in Built Environment 4 (2018) 78.

[5] J. M. W. Brownjohn, Structural health monitoring of civil infrastructure, Philosophical transactions. Series A, Mathematical, physical, and engineering sciences 365 (1851) (2007) 589-622.

[6] H. Wenzel, Health monitoring of bridges, Wiley, Chichester U.K., 2009.

[7] Y. An, E. Chatzi, S.-H. Sim, S. Laflamme, B. Blachowski, J. Ou, Recent progress and future trends on damage identification methods for bridge structures, Structural Control and Health Monitoring 26 (10) (2019) 2297.

[8] A. Deraemaeker, E. Reynders, G. de Roeck, J. Kullaa, Vibration-based structural health monitoring using output-only measurements under changing environment, Mechanical Systems and Signal Processing 22 (1) (2008) 34-56.

[9] F. Magalhães, A. Cunha, E. Caetano, Vibration based structural health monitoring of an arch bridge: From automated OMA to damage detection, Mechanical Systems and Signal Processing 28 (2012) 212-228.

[10] J. M. W. Brownjohn, A. de Stefano, Y.-L. Xu, H. Wenzel, A. E. Aktan, Vibration-based monitoring of civil infrastructure: challenges and successes, Journal of Civil Structural Health Monitoring 1 (3-4) (2011) 79-95.

[11] S. Thöns, M. Döhler, L. Long, On damage detection system information for structural systems, Structural Engineering International 28 (3) (2018) 255-268.

[12] L. Long, M. Döhler, S. Thöns, Determination of structural and damage detection system influencing parameters on the value of information, Structural Health MonitoringIn press (2020).

[13] I. Ojalvo, D. Pilon, Diagnostics for geometrically locating structural math model errors from modal test data, in: 29th Structures, Structural Dynamics and Materials Conference, 1988.

[14] A. Swindlehust, R. Roy, B. Ottersten, T. Kailath, A subspace fitting method for identification of linear state-space models, IEEE Transactions on Automatic Control 40 (2) (1995) 311-316.

[15] G. Gautier, L. Mevel, J.-M. Mencik, R. Serra, M. Döhler, Variance analysis for model updating with a finite element based subspace fitting approach, Mechanical Systems and Signal Processing 91 (2017) 142-156.

[16] M. Friswell, J. E. Mottershead, Finite element model updating in structural dynamics, Vol. 38, Springer Science \& Business Media, 2013.

[17] J. Brownjohn, P.-Q. Xia, H. Hao, Xia. Yong, Civil structure condition assessment by FE model updating: methodology and case studies, Finite Elements in Analysis and Design 37 (10) (2001).

[18] E. Simoen, G. de Roeck, G. Lombaert, Dealing with uncertainty in model updating for damage assessment: A review, Mechanical Systems and Signal Processing 56-57 (2015) 123-149.

[19] É. Balmès, M. Basseville, L. Mevel, H. Nasser, W. Zhou, Statistical model-based damage localization: a combined subspacebased and substructuring approach, Structural Control and Health Monitoring 15 (6) (2008) 857-875.

[20] M. Döhler, L. Mevel, Q. Zhang, Fault detection, isolation and quantification from Gaussian residuals with application to structural damage diagnosis, Annual Reviews in Control 42 (2016) 244-256.

[21] S. Allahdadian, M. Döhler, C. Ventura, L. Mevel, Towards robust statistical damage localization via model-based sensitivity clustering, Mechanical Systems and Signal Processing 134 (2019) 106341. 
[22] M. Döhler, L. Mevel, F. Hille, Subspace-based damage detection under changes in the ambient excitation statistics, Mechanical Systems and Signal Processing 45 (1) (2014) 207-224.

[23] S. Gres, M. D. Ulriksen, M. Döhler, R. J. Johansen, P. Andersen, L. Damkilde, S. A. Nielsen, Statistical methods for damage detection applied to civil structures, Procedia Engineering 199 (2017) 1919-1924.

[24] S. Hoell, P. Omenzetter, Optimal selection of autoregressive model coefficients for early damage detectability with an application to wind turbine blades, Mechanical Systems and Signal Processing 70-71 (2016) 557-577.

[25] M. Basseville, M. Abdelghani, A. Benveniste, Subspace-based fault detection algorithms for vibration monitoring, Automatica 36 (1) (2000) 101-109.

[26] J. Kullaa, Damage detection of the Z24 bridge using control charts, Mechanical Systems and Signal Processing 17 (1) (2003) 163-170.

[27] A. Cabboi, C. Gentile, A. Saisi, From continuous vibration monitoring to fem-based damage assessment: Application on a stone-masonry tower, Construction and Building Materials 156 (2017) 252-265.

[28] N. Cavalagli, G. Comanducci, F. Ubertini, Earthquake-induced damage detection in a monumental masonry bell-tower using long-term dynamic monitoring data, Journal of Earthquake Engineering 22 (sup1) (2018) 96-119.

[29] M. Basseville, L. Mevel, M. Goursat, Statistical model-based damage detection and localization: subspace-based residuals and damage-to-noise sensitivity ratios, Journal of Sound and Vibration 275 (3-5) (2004) 769-794.

[30] É. Balmès, M. Basseville, F. Bourquin, L. Mevel, H. Nasser, F. Treyssède, Merging sensor data from multiple temperature scenarios for vibration-based monitoring of civil structures, Structural Health Monitoring 7 (2) (2008) $129-142$.

[31] D. Straub, Generic approaches to risk based inspection planning for steel structures, Vol. 284, vdf Hochschulverlag AG an der ETH Zurich, 2004.

[32] D. Straub, Probabilistic modeling of non-destructuve testing of steel structures, in: Proceedings of the 4th International PhD Symposium in Civil Engineering, 2002, pp. 311-320.

[33] F. Schoefs, A. Clément, A. Nouy, Assessment of ROC curves for inspection of random fields, Structural Safety 31 (5) (2009) 409-419.

[34] A. Corsano, S. N. Rubin, Detectability of structural failures in offshore platforms by ambient vibration monitoring, in: Proceedings of the Offshore Technology Conference, 1980.

[35] V. Juneja, R. T. Haftka, H. H. Cudney, Damage detection and damage detectability- analysis and experiments, Journal of Aerospace Engineering 10 (4) (1997) 135-142.

[36] K. Worden, J. M. Dulieu-Barton, An overview of intelligent fault detection in systems and structures, Structural Health Monitoring 3 (1) (2004) 85-98.

[37] W.-F. Chen, L. Duan (Eds.), Bridge Engineering Handbook, Second Edition: Seismic Design, CRC Press, Boca Raton, U.S.A., 2014.

[38] J. P. Santos, C. Crémona, A. D. Orcesi, P. Silveira, Multivariate statistical analysis for early damage detection, Engineering Structures 56 (2013) 273-285.

[39] W. Heylen, P. Sas, Modal analysis theory and testing, K. U. Leuven, Belgium, 1997.

[40] P. van Overschee, B. de Moor, Subspace identification for linear systems: theory-implementation-application, Kluwer Academic Publishers, Boston/London/Dordrecht, 1995.

[41] B. Peeters, G. de Roeck, Reference-based stochastic subspace identification for output-only analysis, Mechanical Systems and Signal Processing 13 (6) (1999) 855-878.

[42] A. Benveniste, M. Basseville, G. Moustakides, The asymptotic local approach to change detection and model validation, IEEE Transactions on Automatic Control 32 (7) (1987) 583-592.

[43] E. Parloo, P. Guillaume, M. van Overmeire, Damage assessment using mode shape sensitivities, Mechanical Systems and Signal Processing 17 (3) (2003) 499-518. 
[44] A.-M. Yan, J.-C. Golinval, Null subspace-based damage detection of structures using vibration measurements, Mechanical Systems and Signal Processing 20 (3) (2006) 611-626.

[45] M. D. H. Bhuyan, E. Viefhues, M. Döhler, Y. Lecieux, L. Mevel, F. Hille, F. Schoefs, Output-only subspace and transfer matrix-based damage localization and quantification, in: Proceedings of the 7th International Operational Modal Analysis Conference (IOMAC), 2017.

[46] E. Viefhues, M. Döhler, F. Hille, L. Mevel, Fault detection for linear parameter varying systems under changes in the process noise covariance, in: Proceedings of 21st World Congress of the International Federation of Automatic Control, Berlin, Germany, 2020.

[47] S6-19, Canadian highway bridge design code, Tech. rep., CSA Group, Mississauga Ontario, Canada (2019).

[48] ISO2394, General principles on reliability for structures, Tech. rep., International Organization for Standardization, Geneva, Switzerland (2015).

[49] M. Döhler, L. Mevel, Subspace-based fault detection robust to changes in the noise covariances, Automatica 49 (9) (2013) $2734-2743$.

[50] R. Brincker, C. E. Ventura, Introduction to operational modal analysis, John Wiley \& Sons, 2015. 